\title{
Building Healthier Communities: Value Co-Creation within the Chronic Care Model for Rural Under-Resourced Areas
}

\author{
Christina Higa \\ Communication and Information Sciences \\ University of Hawai $i$ at Manoa \\ christina@uhtasi.org
}

\author{
Elizabeth J. Davidson \\ Shidler College of Business \\ University of Hawai'i at Manoa \\ edavidso@hawaii.edu
}

\begin{abstract}
Chronic disease is a worldwide epidemic that disproportionately affects low- to middle-income countries and regions [1]. The Chronic Care Model (CCM) is intended to address the significant societal costs and health burdens of chronic disease through redesign of the health care system and has raised awareness of the need for integration of clinical services and public health resources. To complement this descriptive, a-theoretical framework, we develop a theory-driven research model rooted in servicedominant logic (S-D logic). Our model conceptualizes improved chronic disease health outcomes as cocreated value and focuses on triadic actor-to-actor-to actor (patients, family/friends and health care providers) resource integration and service exchange. We illustrate the model's utility for policy and intervention design and for research on diabetes selfmanagement programs in low-income, rural communities, in which patients' social capital resources can be integrated with health IT and healthcare expertise in CCM program design.
\end{abstract}

\section{Introduction}

There is a worldwide chronic disease epidemic responsible for 38 million deaths each year [2]. Approximately half of the adults in the United States have one or more chronic disease, [3] accounting for $86 \%$ of America's health care expenditures [4]. This is a considerable cost and health burden. Yet, the majority of premature deaths related to chronic diseases are preventable by individual healthy lifestyle choices such as tobacco and alcohol control, proper nutrition, exercise and medication maintenance.

Traditional health care systems, in part, have contributed to this problem with a focus on acute care. These systems are not equipped nor prepared for addressing systemic life-long chronic conditions. The
Chronic Care Model (CCM) is intended to address these issues through redesign of the health care system. The CCM has had success in raising awareness of the divide between clinical, public health and community interventions by emphasizing that the health care system is a part of the larger community and integration and collaboration among the various actors in this health care ecosystem are essential for addressing the chronic conditions epidemic.

Consistent with this approach, in the U.S. and elsewhere healthcare funding is shifting to value-based policies that emphasize not only care coordination and integrated service delivery but also accountability for improved health outcomes, and thus societal value, from healthcare providers [5]. Health system redesign also includes rebalancing physician-directed activity and patient self-management behaviors. Individual behavior is key in chronic disease management, accounting for nearly $60 \%$ of effects on health whereas the healthcare system accounts for only $10 \%$ [6].

Disparities in societal resources present significant challenges for chronic care management and health system redesign. For instance, social economic status (SES), such as income, education, and social capital, is a driving force of health care disparities; lower SES directly relates to higher levels of disease [7]. As such, chronic disease disproportionately affects low- to middle-income countries and communities [1]. Thus, innovations in health services that capitalize on resources that are available in these communities (such as information technologies) [8], [9] are critical to meeting the growing challenge of chronic diseases.

In this paper, we consider how three types of resources that are (or could be) available in these settings might be integrated to enhance chronic disease management outcomes in low-income, rural communities: (i) the social support of friends and families (a form of social capital); (ii) health information technologies to facilitate service delivery (telehealth); and (iii) health IT to enhance patient engagement in health behavioral changes (e.g., mobile 
smart phones, messaging, and monitors). We develop a conceptual model that draws on service-dominant logic (S-D logic) [10]-[12] to theorize how three types of actors - patients, health-care providers, and family and friends - act as resource integrators to co-create value in terms of reduced individual, social and community burdens of chronic disease. Resources include the expertise of healthcare providers, information technologies for information exchange and communication, and the social capital in friends and family relationships. Our model complements the CCM framework by highlighting the micro-level interactions among actors in co-production of chronic care health services and co-creation of value in health.

In the following sections we review CCM development, contributions, and limitations and our theoretical grounding of co-production of health and co-creation of value guided by S-D logic. We then develop a generalized, theory-driven conceptual model for value co-creation in healthcare and consider how this model could guide the design of chronic disease management interventions in low-income, rural communities. We conclude with a discussion of how this model provides explanatory mechanisms for CCM.

\section{The Chronic Care Model}

The Chronic Care Model (CCM) was developed in the late 1990 s to identify and articulate best practices for addressing the growing burden of chronic conditions in the United States [13]. An Institute of Medicine report published around the same time acknowledged the complexity of this rising problem and suggested that current health care systems were ill equipped and poorly organized to meet the current and emergent health care needs and demands [14]. To this extent, system delivery redesign is a fundamental component of the CCM. Wagner (1999) developed the first iteration, and most referenced, CCM that focuses on the need to integrate patient-centered care and "productive interactions" between patients and health care practice teams to address long-term conditions such as diabetes [15]. The model acknowledges the inadequacy of the traditional primary care system that focuses on treatment of acute illnesses rather than long-term lifestyle changes needed for chronic care management. For instance, clinic visits were characterized as occurring between "uninformed patients" and "unprepared practice teams" [16].

Core elements of the model emphasize six distinct categories for effective chronic condition interventions: (i) improved coordination between health care systems, (ii) community resources, (iii) self-management support, (iv) delivery system design, (v) utilization of decision support tools, and (vi) clinical information systems [14]. The model was empirically driven by literature reviews, a survey of 72 health programs, and input from a 40-member interdisciplinary health expert advisory committee [13].

There are several derivatives of the CCM. In 2002, the World Health Organization (WHO) developed the Innovative Care for Chronic Conditions Framework that expanded the CCM to include a policy framework that strengthens partnerships, includes supportive legislative frameworks, and integrates policy, leadership and advocacy, and financing [17]. The 2002 WHO CCM derivative includes patients' families in a triad of productive interactions with the community and healthcare provider as an added component to Wagner's (1999) model [13].

Barr et al. [18] expanded the CCM to focus with more precision on population health, recognizing the broader determinants of health (e.g., SES) as major barriers to chronic care management. This framework added healthy public policy, supportive environments, and community action as core elements of the community that were not conveyed in the original model. A recent iteration of the CCM is the eHealth Enhanced Chronic Care Model [19], which overlays health information technology systems and services onto Wagner's (1999) CCM. This eHealth model includes emerging communities facilitated by online social networks and mobile applications that connect patients and doctors and that enable access to health information websites and online forums.

The CCM has been used to guide many health care systems and programs with positive outcomes [20], [14] and to raise the awareness of the need for health care system and service redesign. However, as articulated by Wagner [13, pp. 69], "the CCM is not an explanatory theory. It is, like an evidence-based guideline, simply a synthesis of the best available evidence." The WHO (2002), Barr (2003), and Gee (2015) CCM derivatives, while useful, are also descriptive, in most part a-theoretical, and focus on the macro level of the overall healthcare ecosystem. These models represent the overarching health care ecosystem without explaining specifically how or why the component parts work together to achieve improved outcomes. The models' components are descriptive and empirically based, but a-theoretical and assume that if the component parts are in place then positive outcomes will happen, without theorizing the underlying mechanisms through which outcomes arise.

Here, we argue that complementing the policyfocused CCM with a theoretically-informed model that explains "specific pathways by which an intervention ... leads to desired health outcomes" [21, pp. 293] can be used to plan more effective interventions and is of 
particular importance in the complex adaptive healthcare ecosystem with its varied actors, resources, and interactions that are not easily predictable [21]. We suggest a model would be of value that theorizes how patients and health care practice teams have "productive interactions" [15] as they draw from the types of systemic resources outlined in the CCM to improve health outcomes. Such a model should consider patients' knowledge of their disease and its treatment as well as behavioral traits such as selfefficacy and confidence in their ability to manage their condition. A patient's family and friends have a crucial role in managing their chronic conditions [14], as do the providers of healthcare services, suggesting a triad of actor types in such "productive interactions."

\section{Service-dominant logic and health}

The shift towards a healthcare system organized and managed based on value created rather than services provided [22] suggests we draw on theory that would highlight (i) the healthcare ecosystem; (ii) value co-creation that includes patients, families, and providers as essential actors; and (iii) value exchange rather than the performance of particular actions or processes. Research grounded in service-dominant logic [12], [23] is well suited to these goals. In the following sections we introduce the general concepts of co-creation of value in health care services and its roots in service-dominant (S-D) logic as it relates to the main principles of CCM. Secondly we develop a model grounded in S-D logic to more precisely examine "productive interaction" between stakeholders in the health ecosystem. Thirdly, we apply this model to the context of under-resourced, remote, and rural communities to consider how the micro view of "productive interactions" relates to the macro context of the health ecosystem addressed in the CCM.

\subsection{S-D logic and chronic disease management}

Vargo and Lusch's (2004, 2008) seminal works on service-dominant (S-D) logic and co-creation of value introduced a shift in our understanding of the source of value creation and exchange as a producer (firm or organization) delivering value to a consumer (customer, client) by providing products or services. Instead, S-D logic posits a network of actors, operating within an ecosystem of institutional rules, resources, and roles, together they co-create value through service exchange (broadly defined). S-D logic posits that consumers and producers work together for a more customized, user centric service [9]. Traditional linear, dyadic relationships are transformed into dynamic networks of actors and resources [24] in co-production activities. McColl-Kennedy et al. [25, pp. 1] defines value co-creation as "benefit realized from integration of resources through activities and interactions with collaborators in the customer's service network." "Activities" are "performing or doing (cognitive and behavioral)" acts and "interactions" as "ways individuals engage with others in their service network to integrate resources" [23, pp. 6].

In the S-D logic model customers are no longer passive actors but are active members of the ecosystem. This echoes the current focus by both researchers and practitioners in promoting patientcentered care, patient empowerment, and patient selfmanagement [26] approaches to chronic disease management. In this regard there is an emphasis on strong partnerships between the various parts of the health care system and the patient with particular interest in productive interactions as identified in the CCM. More specifically, productive interactions can be viewed as co-production of service and co-creation of value in health care services.

Co-production of health services relates to the activities required to produce a service. Co-creation of value in health services is different because value is not achieved simply because an act is done. Value occurs when the offering is useful to the beneficiary. This is referred to as "value-in-use," in contrast to "value-inexchange" as in the exchange of goods [10], [12]. Value is not created unless all involved actors participate and experience the benefit [10]. This is a key consideration in health care service redesign.

In line with the shift from pay for service to pay for value, the challenge is to effectuate value-in-use and thus value creation, not just to direct the delivery of services. The S-D logic model posits that value is co-created by actor-actor relationships through the integration of resources in activities and interactions between each other. For example, a provider gives a patient with diabetes nutritional information about healthy meals but the patient does nothing with that information (no change in grocery shopping, or meals). In this scenario value to the patient was not achieved and there was no integration of resources. There could be many factors that detract actors from achieving value, for example a language barrier or communication barrier where the information was not presented in the context that the patient could understand. The outcome could be different if the information was re-packaged, say by a Community Health Worker (CHW) attuned to the patient's culture, and delivered in a shared language or culturally appropriate means and with additional information from the patient regarding his or her field of experience, capacities and capabilities. This scenario 
demonstrates the integration of the provider's resources (nutritional information, disease management knowledge), the patient's resources (self- knowledge of their personal situation, routines, etc.) and the CHW's resources (cultural understanding, time in the community), resulting in a newly informed educational offering (integrated resource) that is better tailored to the patient. If the patient acts on this resource to alter their eating habits, co-creation of value is possible. The CHW and patient relationship, integration of information, and shared understanding act as enablers of the co-creation of value.

The value proposition that each actor brings to the exchange varies, as does experienced value. If value exchange is not approached with the intent to co-create shared or overlapping value, resource integration may fail to produce intended outcomes. For example, if a nutritionist was paid for the number of courses she delivered, her end goal might be to deliver as many courses as possible and get paid, regardless of whether the students are informed or motivated by the class to adjust behaviors. Such a scenario within S-D logic does not create value because the value proposition was not meaningful to all the actors involved. Given the shift towards basing reimbursements on value created (and measured) rather than service activities, such outcomes will be detrimental to the health care provider's reputation and reimbursement for services rendered as well (if few patient outcomes improve). Thus we infer from S-D logic that the closer the overlapping of value propositions, there may be more precise alignment of resource sharing, and this will foster co-creation of value. In simple terms, it matters to understand "what's in it" for the individual actors and the overlap of "what's in it" for everyone collectively in order to work together more effectively to create and achieve shared benefits and value.

There are various factors in the roles, activities, and processes that serve as attractors to or detractors from the co-creation of value. These components and interrelationships between actors and resources create a network in which small changes could have large effects [27]. To inform effective restructuring, reconceptualizing and normalizing of CCM processes and structures, an understanding of the mechanisms by which actor interactions lead to value creation is key. We will explore these micro-macro relationships later in this article, although the focus of the proposed model is to address the micro level view of "productive interactions" that underlies the CCM macro view.

\subsection{A2A2A networks in value co-creation}

In this section we articulate a general conceptual model to explain how value is co-created between a triad of health care actors, using S-D logic concepts and proposition. This model provides the generative mechanisms of "productive interactions" between health care providers, patients and family/friends in chronic disease management. Figure 1 depicts the components of our model.

(a) Actors: In Figure 1, "A" represents an actor type. Actors draw on and activate resources, which they access from their own stores or from the service ecosystem; actors are resource integrators [11]. The CCM has been focused primarily on the patientprovider relationships. The A2A2A S-D logic model introduces a triadic relationship of the actors rather than the A2A dyadic models common to S-D logic research, though potentially there could be any number of actors that serve as resource integrators.

(b) [Integrated] Resources: In Figure 1 resources are represented by the shaded, dotted circle around the actors as part of the shared resource pool in the ecosystem or provided by a service platform [9]. The dotted rectangle represents resources each actor can control and activate. Vargo and Lusch [10], [12] define resources as anything an actor can draw on for support. Operant resources are characterized as intangible, infinite and dynamic such as information and knowledge, and information technology [10]. Operand resources are tangible, finite, and static such as natural resources, equipment, and goods [11]. As more actors join the network, and as operant resources are used to act on operand resources, the resource pool exponentially increases. This S-D logic view shifts the emphasis of resource limitations in CCM. For instance, using telehealth in chronic care activities potentially leverages (expands the reach) of the provider's knowledge resources.

(c) Interactions: McColl-Kennedy et. al. [25] describe interactions as ways individuals engage with others in their service network to integrate resources. The CCM articulates the need to improve interactions between uninformed patients and ill-prepared providers with specific tools like patient self-management practices, decision support tools for providers and clinical information systems. However, service exchange and resource integration interactions draw from or develop into institutionalized roles, rules, and practices, some of which may be governed by a service platform provider [9]. Thus service ecosystem change is necessarily institutional change. Various instantiations of the CCM have outlined institutional change at the service platform level (e.g., revised policies for health delivery system) but provides little insight into the micro-level interactions in which institutional change actually occurs (or does not).

(d) Activities: McColl-Kennedy et. al. [25] describe activities as performing or doing cognitive 
and behavioral acts. These activities occur in the context of actor-to-actor interactions and actor resource integration. As it had been applied to a general, macro level of health delivery system, the CCM provides little insight into specific activities beyond the need for coordination among various system actors (such as inoffice and hospital care givers) and "productive interactions" between them. Evidence-based medicine care protocols are knowledge-based resources for healthcare providers to inform their patients. However, S-D logic highlights that without proper integration and alignment, value-in-use is not likely.

(e) Value: Value is defined as "...dynamic, experiential and contextual, rather than a unit of output or an embedded property of a good or service" [6, pp. 143]. Each actor may derive different types of value from an exchange, though value is additive, so that the viability of the system as a whole is enhanced. "Value" in health and healthcare is hard to define and difficult to assess. With the movement to value-based reimbursement for healthcare service providers, tangible measures like reductions in $\mathrm{A} 1 \mathrm{C}$ values (for diabetic control) or patient satisfaction scores are rapidly gaining hold as proxies for value.

(f) Co-Creation of Value: A core tenet of S-D logic is that value is co-created though actors' activities and resource integration in the ecosystem, and it occurs when a service offering is used and benefits the recipient. Value-in-use [10], [12] is represented by the arrows pointing to the actors from the circle center in Figure 1. In the CCM model, cocreated value implies improved health outcomes for individuals, lower health system costs within care delivery systems, and more productive and satisfied communities (and society).

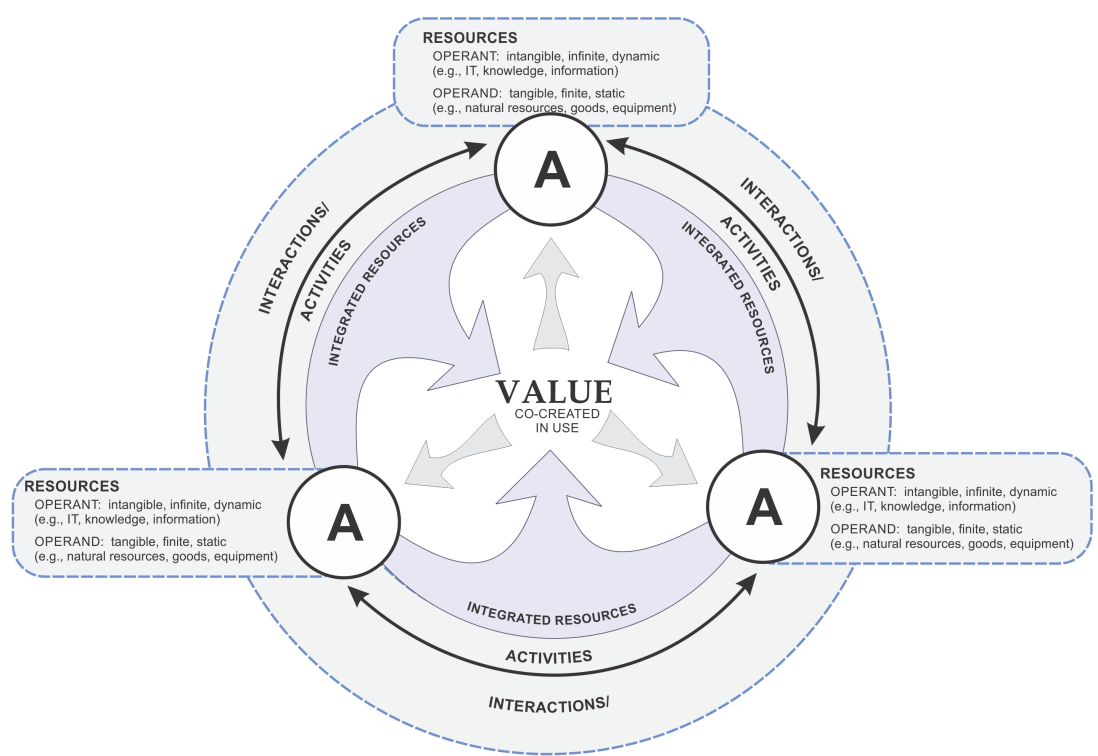

Figure 1. A2A2A S-D logic model

\section{Chronic disease management in under- resourced, rural settings}

Our research goal is to develop and apply a conceptual model that can explain theoretically the mechanisms through which resources applied in chronic disease management activities may be effective and to inform the design of interventions and research into these programs. Here, we illustrate how the general conceptual model above may be applied. Our context is management of diabetes in a community with low social economic status (SES) in terms of rurality and low-income, but very high social support.

Challenges in improving chronic conditions are compounded in low SES communities because there is a persistent relationship between SES and chronic disease and mortality [28]. SES includes social and environmental factors that are not easily manipulated or corrected. Thus, due to the systemic nature of the problem, low SES in relation to poor health is often ignored and this adversely impacts the high cost and health burden of chronic disease in general but more intensely in small, rural and low-income communities. Thus, health services innovation is especially important for communities with poor SES that are seeking to leverage their strengths and maximize limited resources [8]. However instead of focusing on the overwhelming challenges, it is more productive to build on the 
community strengths and opportunities. The strength of social connections in such settings present community resources that might contribute to the community's and individuals' ability to respond to chronic disease health care needs. IT is potentially critical for rural communities with scarce resources because IT can enhance communication and connections to increase access to knowledge, information, and services not readily available within these local communities. Our application of the A2A2A S-D logic model reflects the dynamics of social support and application of IT to facilitate resource integration among actors.

\subsection{Applying the A2A2A S-D logic model}

Figure 2 depicts this applied model focused on three types of actors: patient, family and friends, and health care provider. Family and friends add one additional node to the traditional dyadic focus between the patient and provider. Diabetes should be considered a family rather than individual disease [29]. Family members play a critical role in daily routines and with proper education and training can contribute to improvements in patient self-efficacy, perceived social support, diabetes knowledge and self-care activities [30]. "Using a family lens when addressing diabetes management may contribute to more effective practices and better outcomes for all involved" [28, pp. 51].

Diabetes management is complex because it involves understanding, monitoring, and balancing several interrelated effects such as blood glucose levels, nutrition (carbohydrate and sugar intake), exercise, and medication. Resources include the expertise of the healthcare providers and their information and knowledge relating to the etiology of diabetes and the personal and contextual information about the family. Social support in family/friend relationships and IT resources (e.g., Bluetooth-enabled blood glucose monitors, mobile phones, and telehealth services) are operant resources depicted in our model, which also includes operand resources such as medicine, medical supplies, goods and equipment.

Capitalizing on resources such as social capital available in small communities is critical to meeting the growing challenges of chronic conditions. The World Health Organization identifies social capital as a key element for creating health equity and wellbeing of individuals and communities [2]. Some influencing elements include social networks that provide intimacy, companionship, and resources for coping with illness, information for understanding illness and treatment, and support for dealing with stress. House's [31] seminal work in social capital provides further description in four general categories of social support including emotional, instrumental, informational, and appraisal. Studies have demonstrated that social support can improve patient engagement and health outcomes for patients with chronic illness [32]-[34]. Thus the model considers how adding an actor type (family and friends) brings new resources to the available pool of resource that can be integrated to co-create value. Specific activities may include providing social support to assist the patient with medication adherence, blood glucose monitoring, and other routines such as meal planning to improve patient diabetes self-management [29], [30], [35]-[37].

However, in order to be effective with patient support activities (reminders, meal planning, blood glucose monitoring) family members must be informed. Including family members in educational interventions is not a norm in patient self-management education but could potentially prepare families to more effectively provide emotional and psychological support to patients with diabetes [38]. Health care providers, patients, and family/friends integrate information, knowledge, and experiences to enhance and better inform diabetes education classes, patient health plans and goals.

\subsection{IT resources in the A2A2A S-D logic model}

The role of IT in health care is central in establishing a value network for sharing and integrating information and resources [9], [10], [39], [40]. Key health organizations in the U.S. including the Institute of Medicine and the Agency for Healthcare Research and Quality (AHRQ) recommend the use of IT for supporting self-management of chronic conditions [19]. More specifically mobile technologies, such as text messaging, increase patient behavior and self-efficacy in diabetes self-management [41]-[43]. Common measures used in such studies follow the American Diabetes Association recommendations for self-care, including medication adherence, foot care, and blood sugar monitoring. Further, studies that focus on the use of text messaging for patient and friends/family support [43], [44] have found initial positive impacts in the use of text messaging and patient support systems in both self-efficacy and medical measures.

Text messaging can be used for enhanced and more frequent communication between the actors to fill the communication gap between clinic visits. Hussein, Hasan, Jaradat's [45] study found that patient contact, via text messaging, with providers or diabetes educators significantly reduces HbgA1c levels by $1.16 \%$ lower than control groups. Dick, et al.'s [41] study, that sent personalized messages regarding medication, foot care and appointments, showed a decrease in missed medication doses by 1.6 per week to .6 and increased patient confidence in diabetes self-management. Bluetooth-enabled blood glucose (BG) monitors and 
mobile phone applications enable patients to more conveniently check blood levels and transmit results to an online database accessible by all actors. Family/friends and health care providers are able to see the patient activities (number of times BG was checked and levels reported). This informs family/friends and health care providers and enables more efficient support to the patient (when patients need reminders to take a $\mathrm{BG}$ reading, or check-in on high $\mathrm{BG}$ reports). Telehealth is another key resource especially for severely resource constraint communities where there are lack of specialists and education and training opportunities. Telehealth services can help to facilitate bridging outside resources necessary for making improvements in chronic care management [46], [47], reduce spending and improve clinical measures [47]. The video teleconferencing equipment (operand resource) enables the access to knowledge (operant resource) and services of certified diabetes educators, nutritionists, endocrinologists, and psychologists not available in the local community. The connection to these health care providers (actors) increases the resource pool available to the patient, family/friends, and community (actors) and increases the opportunities for resource integration and co-creation of value.

\subsection{Value and value co-creation}

The applied model presents a scenario where the shared value propositions are patient controlled diabetes and respectively a healthier patient, family, and health care system. This model highlights that value is achieved when the offering is received, used, and is of benefit to the actors. That is, value arises when the integration of information, knowledge, and social support facilitated by IT results in improved and tailored diabetes education is not only delivered by the health care provider but also received and put into action by the patient and family/friends. This in turn results in healthier lifestyle choices (supported by change in food selection and meals, improved self-management activities such as BG readings, medication adherence, etc.), that ultimately leads to improved clinical measures for the patient (lower A1C, lower BG) resulting in the targeted co-created value. Each actor may have engaged in resource exchange interactions with differing individual priorities and propositions of value (e.g., health care provider ultimately wants to earn an income, family/friends want less medical bills, patient wants to have more energy); however, to achieve this we infer from a S-D logic perspective, that shared or overlapping value propositions are needed to realize co-created value involving all actors.

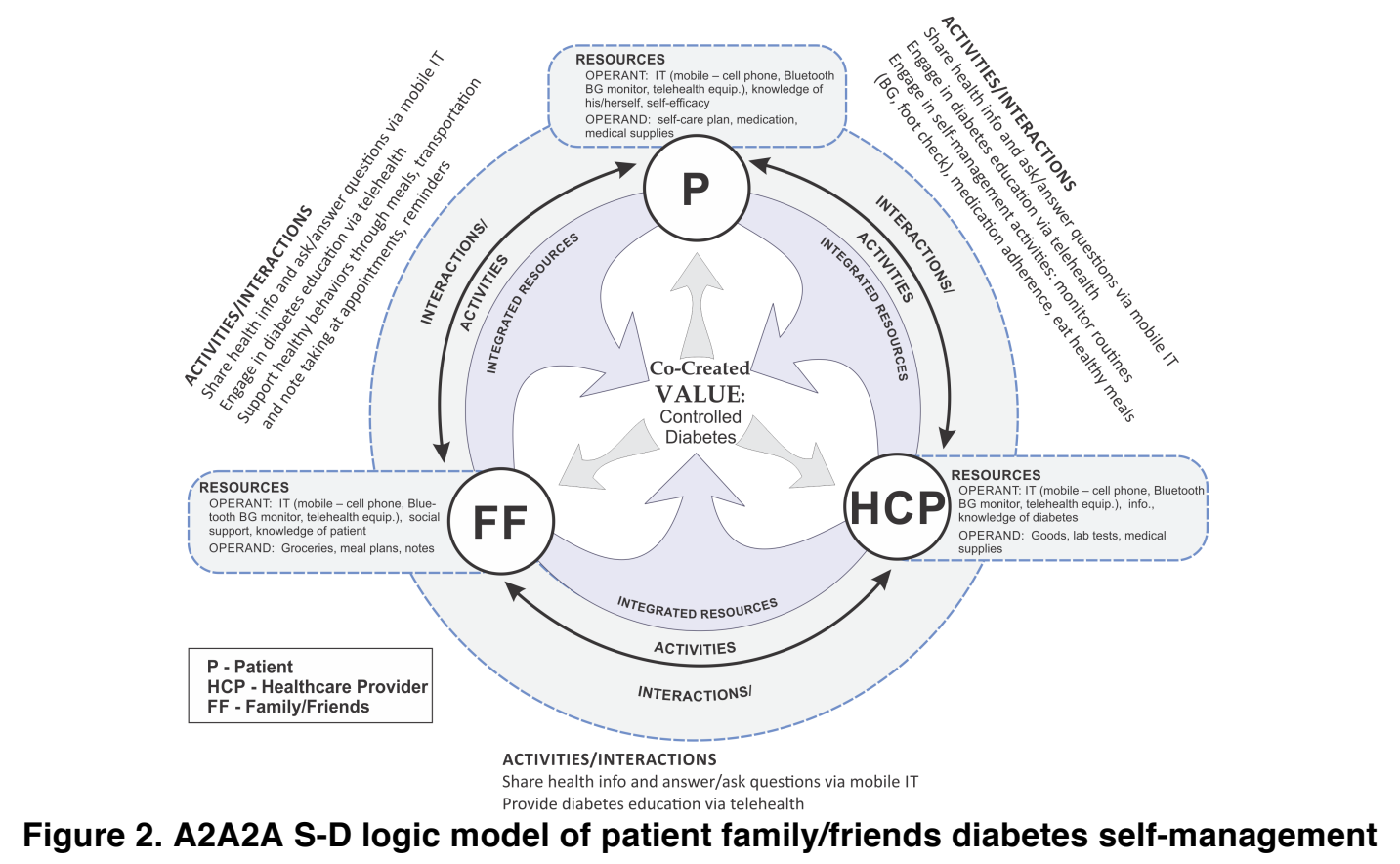

\section{From micro-to-macro CCM}

Our micro-level A2A2A S-D logic model foregrounds the "productive interactions" that underlie the CCM. This conceptual model, derived from the principles of service dominant logic, assumes that the right actors are willing and available to engage in resource integration and exchange that can lead cocreated values of improved individual and community health. It also assumes that the resources needed are 
accessible, and can be amplified (made more productive) through resource integration. Admittedly this is an optimistic depiction of the possibility for population-wide chronic disease management, particularly in under resourced communities. We acknowledge that patients, family/friends and health care providers are situated in informal and formal institutions within the larger context of healthcare ecosystems that both constrain and enable their productive interactions. As the various CCM versions have articulated, resources are unevenly distributed and are made harder to access by lack of coordination between various healthcare system actors and components. No one "service platform" [9] governs all of the institutionalized roles, practices, rules and resources that could contribute to addressing this worldwide health system epidemic.

Improvements depend on institutional changes that are tough to bring about as well as technological and social innovation. We suggest that S-D logic overall, and our conceptual A2A2A model, highlight fresh thinking to do so. First, S-D logic recognizes that institutions are needed to orchestrate the various actors, resources and their interactions. Innovative new relationships and processes require effort to develop, refine, adapt, and modify. Once these processes are more established (institutionalized) actors transition into a maintenance mode that is routinized and requires less energy to maintain. In this transition from 'new to established' a set of institutional principles and structures to facilitate resource integration and the processes described in the A2A2A S-D logic model are needed [9] [10]. However, we suggest that principles and structures do not arise solely (or even primarily) from top-down policy initiatives, as we typically see in versions of the CCM. Institutions arise from actors' day-to-day interactions and innovation from their experimentation. The A2A2A conceptual model highlights opportunities to investigate these interactions: (i) Are the right actors involved in a valueseeking service exchange scenario? (ii) What other actors may be available, and how can their contribution (of resources and of activities) be usefully included? (iii) What are the obstacles these actors face accessing and integrating resources, and can obstacles be reduced or eliminated by bringing new operant resources (such as health IT applications) into a resource pool? (iv) What resources may be present in the ecosystem but not yet recognized (such as the social capital of patients expressed via social support)? (v) Are value propositions sufficiently aligned so that co-production of a health service results in value-in-use for all actors and co-creation of value is possible?

For example, the health care system (a formal institution) and the family/friends (an informal institution) overlap in the relationships between the patient, family/friends and health care provider. The value of and opportunities to include family and friends into chronic care management programs are relatively new, experimental approaches. Barriers exist to allowing these new actors to engage fully in service coproduction and value co-creation processes, such as privacy laws that may limit health information sharing or healthcare providers' discomfort with including others into their patient interactions (aside from caregiver situations such as children and parents or aged parents and children). Top-down policy mandates alone may do little to overcome these barriers, and may even fail to acknowledge their impact. The A2A2A S-D logic model shines theoretical light on such issues.

\section{Concluding remarks}

Chronic conditions are among the most common, costly, and preventable diseases. Yet, the mortality rates for chronic disease in the United States and worldwide are staggering. Although there is increased urgency to identify effective strategies to overcome this epidemic, solutions are complex because of the systemic nature of the conditions that are impacted, in large part, by lifestyle choices for individuals in communities and health ecosystems. The CCM is intended to address the significant societal costs and health burdens of chronic disease through redesign of the health care system with a focus on the integration of clinical services and public health resources. The CCM provides an overarching and high-level view of core elements for effective chronic condition interventions to guide top-down policies and system redesign, but these models are descriptive and a-theoretical; they assume but do not explain the mechanisms that lead to positive outcomes. While CCM models have informed health care policy, the escalating worldwide epidemic of chronic diseases indicates that more theoretical and applied work is needed.

Here we develop a theory-informed model that supports the CCM by adding an explanatory framework that conceptualizes chronic disease and health outcomes as co-created value among actors in the health ecosystem. Our model is rooted in S-D logic and supports the CCM by: (i) theorizing how "patient engagement" operates through interactions among three types of actors - patients, health-care providers, and family and friends, (ii) drawing research and policy attention beyond simply the lack of resources and lack of coordination, to the mechanisms for resource integration realized through actors' activities and interactions, (iii) focusing on co-created value as the ultimate goals, in terms of reduced individual, social 
and community burdens of chronic disease, and the related enablers and barriers, and (iv) highlighting innovations possible when new actors (family and friends) and new resources (social capital, IT) are recognized within the ecosystem.

Our conceptual model will be useful to inform intervention and action research projects. For instance, we are not aware of any studies that have yet investigated the co-creation of health value specifically in relationship to support from family and friends and IT interventions. More information is needed to understand this phenomena in terms of quality, extent of family participation, and outcomes [30], [48]. The conceptual model per se is not suitable for theory or hypothesis testing. However, testable hypothesis for specific projects to investigate questions such as those we identified earlier (e.g., Can including a new actor increase realization of value-in-use? Do certain factors inhibit or increase resource integration?) are feasible. Such research can draw on well-established measures (A1C and blood glucose levels, adherence to patient self-monitoring routines regarding nutrition, exercise and medication) as proxies for value co-creation, though we would caution that such measurements are simply proxies and are not tapping into value per-se. Better understanding of the types of value that actors realize in chronic disease management programs may require more in-depth and situated study. Finally, we hope that the proposed model helps to facilitate the operationalization of the CCM, developed over a decade ago and yet its core principles are still essential in today's health care environment.

\section{References}

[1] World Health Organization, "Non Communicable Diseases, 2015," 2015. [Online]. Available: http://www.who.int/mediacentre/factsheets/fs355/en/. [Accessed: 10-Jun-2016].

[2] World Health Organization, "A Conceptual Framework for Action on the Social Determinants of Health," Geneva, 2010.

[3] B. Ward, J. Schiller, and R. Goodman, "Multiple Chronic Conditions Among US Adults: A 2012 Update," Prev. Chronic Dis., 2014.

[4] J. Gerteis, D. Deitz, L. LeRoy, R. Ricciardi, T. Miller, and J. Basu, "Multiple Chronic Conditions Chartbook 2010 Medical Expenditure Panel Survey Data," 2010.

[5] S. M. Burwell, "Setting Value-Based Payment Goals HHS Efforts to Improve U.S. Health Care," N. Engl. J. Med., Mar. 2015, vol. 372, no. 10, pp. 897-899.

[6] J. M. McGinnis, P. Williams-Russo, and J. R. Knickman, "The Case for More Active Policy Attention to Health Promotion," Health Aff., 2002, vol. 21, no. 2, pp. 78-93.

[7] B. G. Link and J. Phelan, "Social Conditions as Fundamental Causes of Disease.," J. Health Soc. Behav., Jan. 1995, vol. Spec No, pp. 80-94.
[8] M. Barrett, E. Davidson, J. Prabhu, and S. L. Vargo, "Service Innovation in the Digital Age: Key Contributions and Future Directions," MIS Q., 2015, vol. 39, no. 1, pp. 134-154.

[9] S. Srivastava and G. Shainesh, "Bridging the Service Divide Through Digitally Enabled Service innovations; Evidence from Indian Health Care Service Providers," MIS Q., 2015, vol. 39, no. 1.

[10] R. F. Lusch and S. Nambisan, "Service Innovation: A Service-Dominant Logic Perspective," MIS Q., 2015, vol. 39, no. 1, pp. 155-175.

[11] S. L. Vargo and R. F. Lusch, "Evolving to a New Dominant Logic for Marketing," J. Mark., 2004, vol. 68, no. 1, pp. 1-17.

[12] S. L. Vargo and R. F. Lusch, "Service-dominant Logic: Continuing the Evolution," J. Acad. Mark. Sci., 2008, vol. 36 , no. 1, pp. 1-10.

[13] E. H. Wagner, C. Davis, J. Schaefer, M. Von Korff, and B. Austin, "A Survey of Leading Chronic Disease Management Programs," Illness/Disease Manag., 1999, vol. 02 , pp. 10.

[14] E. H. Wagner, B. T. Austin, C. Davis, M. Hindmarsh, J. Schaefer, and A. Bonomi, "Improving Chronic Illness Care: Translating Evidence Into Action," Health Aff., Nov. 2001, vol. 20, no. 6, pp. 64-78.

[15] A. Collinsworth, M. Vulimiri, C. Snead, and J. Walton, "Community Health Workers in Primary Care Practice: Redesigning Health Care Delivery Systems to Extend and Improve Diabetes Care in Underserved Populations," Health Promot. Pract., Nov. 2014, vol. 15, no. 2 Suppl, pp. 51S-61S.

[16] T. Bodenheimer, E. H. Wagner, and K. Grumbach, "Improving Primary Care for Patients with Chronic Illness.," JAMA, Oct. 2002, vol. 288, no. 14, pp. 17751779.

[17] R. Beaglehole, J. Epping-Jordan, V. Patel, M. Chopra, S. Ebrahim, M. Kidd, and A. Haines, "Improving the Prevention and Management of Chronic Disease in LowIncome and Middle-Income Countries: A Priority for Primary Health Care," Lancet, London, England, Sept. 2008, vol. 372, no. 9642, pp. 940-949.

[18] V. J. Barr, S. Robinson, B. Marin-Link, L. Underhill, A. Dotts, and D. R. and S. Salivaras, "The Expanded Chronic Care Model: An Integration of Concepts and Strategies from Population Health Promotion and the Chronic Care Model," Healthc. Q., Nov. 2003, vol. 7, no. 1.

[19] P. M. Gee, D. A. Greenwood, D. A. Paterniti, D. Ward, and L. M. S. Miller, "The eHealth Enhanced Chronic Care Model: A Theory Derivation Approach," Journal of Medical Internet Research, Apr. 2015, vol. 17, no. 4. pp. e86.

[20] M. Stellefson, K. Dipnarine, and C. Stopka, "The Chronic Care Model and Diabetes Management in US Primary Care Settings: A Systematic Review," Prev. Chronic Dis., Jan. 2013, vol. 10, pp. E26.

[21] N. Freudenberg, E. Eng, B. Flay, G. Parcel, T. Rogers, and N. Wallerstein, "Strengthening Individual and Community Capacity to Prevent Disease and Promote Health: In Search of Relevant Theories and Principles.," Health Educ. Q., Aug. 1995, vol. 22, no. 3, pp. 290-306. 
[22] M. E. Porter, "A Strategy for Health Care Reform Toward a Value-Based System," N. Engl. J. Med., Jul. 2009, vol. 361, no. 2, pp. 109-112.

[23] S. L. Vargo and R. F. Lusch, "The Four Service Marketing Myths: Remnants of a Goods-Based, Manufacturing Model," 2004, J. Serv. Res., vol. 6, no. 4, pp. 324-335.

[24] A. Honka, K. Kaipainen, H. Hietala, and N. Saranummi, "Rethinking Health: ICT-Enabled Services to Empower People to Manage Their Health.," IEEE Rev. Biomed. Eng., Jan. 2011, vol. 4, pp. 119-139.

[25] J. R. McColl-Kennedy, S. L. Vargo, T. S. Dagger, J. C. Sweeney, and Y. V. Kasteren, "Health Care Customer Value Cocreation Practice Styles," J. Serv. Res., May 2012, vol. 15, no. 4, pp. 370-389.

[26] R. Palumbo, "Contextualizing Co-Production of Health Care: A Systematic Literature Review," Int. J. Public Sect. Manag., 2016, vol. 29, no. 1, pp. 72-90.

[27] P. E. Plsek and T. Wilson, "Complexity, Leadership, and Management in Healthcare Organisations.," BMJ, Sep. 2001, vol. 323, no. 7315, pp. 746-749.

[28] J. C. Phelan, B. G. Link, and P. Tehranifar, "Social Conditions as Fundamental Causes of Health Inequalities: Theory, Evidence, and Policy Implications.," J. Health Soc. Behav., Jan. 2010, vol. 51 Suppl, no. 1_suppl, pp. S28-40.

[29] S.A. Denham, M. M. Manoogian, and L. Schuster, "Managing Family Support and Dietary Routines: Type 2 Diabetes in Rural Appalachian Families," Fam. Syst. Heal., 2007, vol. 25, no. 1, pp. 36-52,.

[30] A.A. Baig, A. Benitez, M. T. Quinn, and D. L. Burnet, "Family Interventions to Improve Diabetes Outcomes for Adults.," Ann. N. Y. Acad. Sci., Sept. 2015, vol. 1353, pp. 89-112.

[31] J. S. House, Work Stress and Social Support. AddisonWesley: Reading, Mass., 1981.

[32] R. M. Scheffler, T. T. Brown, L. Syme, I. Kawachi, I. Tolstykh, and C. Iribarren, "Community-Level Social Capital and Recurrence of Acute Coronary Syndrome.," Soc. Sci. Med., Apr. 2008, vol. 66, no. 7, pp. 1603-1613.

[33] J. Strom and E. LE, "The Impact of Social Support on Outcomes in Adult Patients with Type 2 Diabetes: A Systemic Review," Curr. Diab. Rep., 2012, vol. 12, no. 6, pp. $769-781$.

[34] E. J. Nicklett and J. Liang, "Diabetes-related Support, Regimen Adherence, and Health Decline Among Older Adults.," J. Gerontol. B. Psychol. Sci. Soc. Sci., May 2010, vol. 65B, no. 3, pp. 390-399.

[35] T.A. Armour, S. L. Norris, L. Jack, X. Zhang, and L. Fisher, "The Effectiveness of Family Interventions in People with Diabetes Mellitus: A Systematic Review.," Diabet. Med., 2005, vol. 22, no. 10, pp. 1295-1305.

[36] J. Gleeson-Kreig, H. Bernal, and S. Woolley, "The Role of Social Support in the Self-Management of Diabetes Mellitus Among a Hispanic Population," Public Health Nurs., 2002, vol. 19, no. 3, pp. 215-222.

[37] J. Hu, K. Amirehsani, D. C. Wallace, and S. Letvak, "Perceptions of Barriers in Managing Diabetes: Perspectives of Hispanic Immigrant Patients and Family Members," Diabetes Educ., 2013, vol. 39, no. 4, pp. 494-503.
[38] J. Hu, D. C. Wallace, T. P. McCoy, and K. A. Amirehsani, "A Family-Based Diabetes Intervention for Hispanic Adults and Their Family Members," Diabetes Educ., 2013, vol. 40, no. November, pp. 48-59.

[39] S. Stewart Loane, C. M. Webster, and S. D'Alessandro, "Identifying Consumer Value Co-created through Social Support within Online Health Communities," $J$. Macromarketing, May 2014, vol. 35, no. 3, pp. 353-367.

[40] P. Nambisan and S. Nambisan, "Models of Consumer Value Cocreation in Health Care," Health Care Manage. Rev., 2009, vol. 34, no. 4, pp. 344-354.

[41] J. J. Dick, S. Nundy, M. C. Solomon, K. N. Bishop, M. H. Chin, and M. E. Peek, "Feasibility and Usability of a Text Message-based Program for Diabetes Selfmanagement in an Urban African-American Population.," J. Diabetes Sci. Technol., Sept. 2011, vol. 5, no. 5, pp. 1246-1254.

[42] S. Hamine, E. Gerth-Guyette, D. Faulx, B. B. Green, and A. S. Ginsburg, "Impact of mHealth Chronic Disease Management on Treatment Adherence and Patient Outcomes: A Systematic Review," J. Med. Internet Res., Feb. 2015, vol. 17, no. 2, pp. e52.

[43] D. W. Roblin, "The Potential of Cellular Technology to Mediate Social Networks for Support of Chronic Disease Self-management.," J. Health Commun., 2011, vol. 16 Suppl 1, no. May 2012, pp. 59-76.

[44] J. E. Aikens, K. Zivin, R. Trivedi, and J. D. Piette, "Diabetes Self-management Support Using mHealth and Enhanced Informal Caregiving," J. Diabetes Complications, 2014, vol. 28, no. 2, pp. 171-176.

[45] W. I. Hussein, K. Hasan, and A. A. Jaradat, "Effectiveness of Mobile Phone Short Message Service on Diabetes Mellitus Management: The SMS-DM Study," Diabetes Res. Clin. Pract., 2011, vol. 94, no. 1.

[46] L. C. Baker, S. J. Johnson, D. Macaulay, and H. Birnbaum, "Integrated Telehealth and Care Management Program for Medicare Beneficiaries with Chronic Disease Linked to Savings," Health Aff. (Millwood)., Sept. 2011, vol. 30, no. 9, pp. 1689-1697.

[47] S. Shea, R. S. Weinstock, J. A. Teresi, W. Palmas, J. Starren, J. J. Cimino, A. M. Lai, L. Field, P. C. Morin, R. Goland, R. E. Izquierdo, S. Ebner, S. Silver, E. Petkova, J. Kong, and J. P. Eimicke, "A Randomized Trial Comparing Telemedicine Case Management with Usual Care in Older, Ethnically Diverse, Medically Underserved Patients with Diabetes Mellitus: 5 Year Results of the IDEATel Study.," J. Am. Med. Inform. Assoc., Jan. 2009, vol. 16, no. 4, pp. 446-456.

[48] A. M. Rosland, E. Kieffer, B. Israel, M. Cofield, G. Palmisano, B. Sinco, M. Spencer, and M. Heisler, "When is Social Support Important? The Association of Family Support and Professional Support with Specific Diabetes Self-Management Behaviors," J. Gen. Intern. Med., 2008, vol. 23, no. 12, pp. 1992-1999. 